\title{
Concrete pavements management
}

\author{
Łukasz Rudziński ${ }^{1,2}$ Mieczysław Stowik ${ }^{3}$ \\ ${ }^{1}$ Autostrada Wielkopolska II SA, Dziadoszańska 10 Poznań, Poland \\ ${ }^{2}$ Doctoral student, Poznan University of Technology, Piotrowo 5 Poznań, Poland \\ ${ }^{3}$ Poznan University of Technology, Institute of Civil Engineering, Piotrowo 5 Poznań, Poland
}

\begin{abstract}
The article discusses the issue of managing roads with concrete surfaces. Modern methods of surface features identification applied on the exploited motorway section of a length of $100 \mathrm{~km}$ (in conversion to rows of concrete slabs it gives around $600 \mathrm{~km}$ ) are presented. The general principles of the rigid surface management system are described below in order to enable a comprehensive analysis of the obtained data in terms of the possibility of developing concrete slabs degradation models that form the basis for long-term planning of maintenance works.
\end{abstract}

\section{Introduction}

Portland cement concrete is becoming increasingly used as a road paving material in many projects on a number of key sections of the major highways of the Polish road network (motorways and trunk roads). This makes appropriate maintenance a key issue, so that the design lifetime of 30-40 years could be achieved with minimum maintenance requirement in the first phase of pavement life [1].

Contemporary pavement distress evaluation methods, including in particular surface conditions identification techniques are not commonly known in Poland and should be popularised in view of the increasing number of roads with concrete pavements and prepared (designed to accommodate different concrete slab geometries with the slab width exceeding the width of the traffic lane) to ensure the required quality of the road network data without compromising the efficiency and maintaining the non-intrusive nature of the pavement condition surveys. An important part of the pavement management process during its service life is appropriate monitoring of the pavement condition consisting in collecting condition

\footnotetext{
${ }^{1}$ Corresponding author: lukasz.k.rudzinski@,doctorate.put.poznan.pl
} 
evaluation input data which are critical for reliable residual life modelling. This knowledge is indispensable for planning the maintenance treatments and renewals, defining priorities and allocation of funds in the long term. The necessary inputs for pavement condition modelling include evaluation of its current condition. Together with the identification of the causes of distress it is the basis for making pavement condition projections. Failure to carry out maintenance treatments at low severity level of distress at an appropriate time can considerably increase the cost of maintenance necessary to repair seriously deteriorated pavement. Figure 1 shows typical concrete pavement curves presenting the periods in which individual treatments and curves extending the life cycle of the pavement are performed through appropriate management of maintenance procedures.

The aim of the presented research work is to develop a solution that allows obtaining the longest life cycle of a concrete surface by analyzing surface images of individual concrete slabs and using them to plan maintenance treatments.

a)

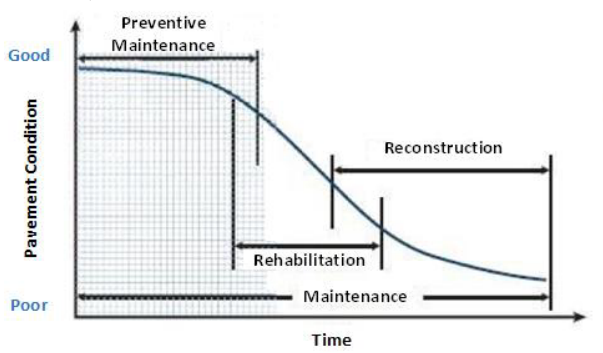

b)

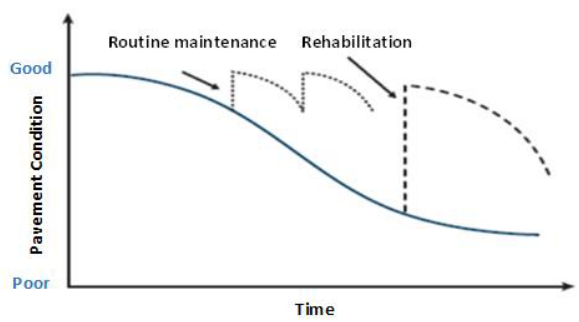

Fig.1. Typical concrete pavement curves presenting the periods in which individual treatments are performed (a) and curves extending the life cycle of the pavement through appropriate management of maintenance procedures (b) [2]

\section{Contemporary identification method of the surface conditions of concrete pavements}

In road diagnostics we can speak of a successful introduction of equipment or technologies no sooner than after they have been verified under traffic [3]. For this reason, in this presentation of the contemporary surface conditions identification technique its reliability has been verified on a ca. $100 \mathrm{~km}$ long section of concrete pavement with dowelled and tied joints (ca. $600 \mathrm{~km}$ total length of concrete lanes) consisting of the total number of 121,185 slabs of $4 \times 5 \mathrm{~m}$ and $3 \times 5 \mathrm{~m}$ in size.

Bearing in mind that a contemporary distress evaluation method should be based on repeatable measurements and taking into account errors resulting from different readings of the on-board GPS and distance measuring devices (in the range of up to 10 or 20 metres) between different surveys (which are, however, acceptable for most maintenance management procedures) it is necessary to assign each detected distress to a specific slab. To this end a dedicated slab layout map has been prepared including appropriate referencing of the pavement slabs. All the concrete slabs have been identified with $\mathrm{x}$, y co-ordinates and a unique ID ensuring repeatability relating 
the respective slabs and enabling their prompt and definite identification by the maintenance services assisted with Trimble system (Fig. 2). This ensured repeatability of measurements at the slab level. This approach is necessary to ensure synchronised data of appropriate quality that can be used for further verification of the condition of pavement. It also supports detailed life-cycle analyses at the level of individual slabs rather than over a dozen metres long road segments. Moreover, with this method of identification it is possible to avoid interfering with the equipment installed on the motorway.
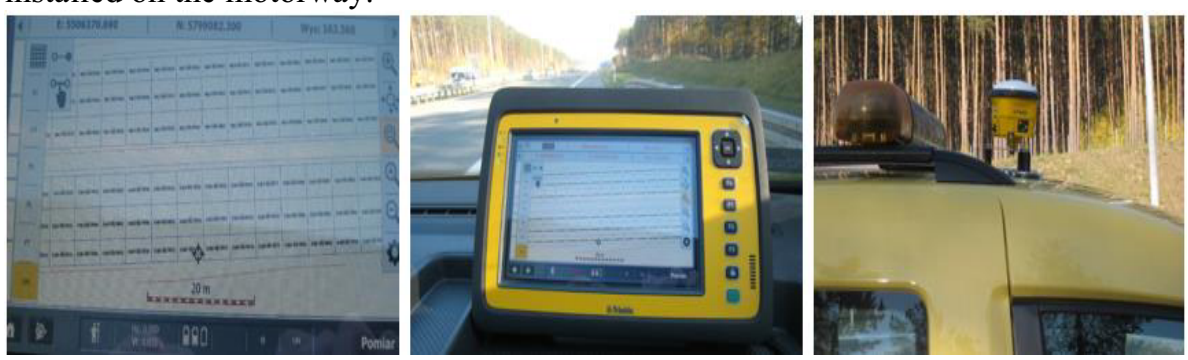

Fig. 2. Slab identification kit

As a part of the conducted diagnostics of the concrete pavement, a research methodology was developed, using a special vehicle recording surface conditions of the pavement. The pavement was surveyed with the System for Pavement Distress Evaluation (SPDE) at the disposal of the Polish Road and Bridge Research Institute (IBDiM) (Fig. 3a). SPDE provides digital images of the pavement surface and any distress it contains over up to $3.9 \mathrm{~m}$ wide strip with $1 \mathrm{~mm} \times 1 \mathrm{~mm}$ image resolution. It was designed to enable surveying road network in any traffic conditions. LED lighting technology makes the technique insensitive to any variation of lighting conditions. Flashes of LED lights have a high magnitude in the recorded spectrum, several times the daylight level. There is also a right-of-way camera installed in the front of the lane.

The images obtained with this special equipment were additionally processed and calibrated to enable geometrical measurements of the identified distresses. This was a necessary step to ensure accurate identification of both surface and subsurface types of distress.

Image calibration was performed using affine transformations and image filtering and pattern recognition algorithms were used to detect transverse and longitudinal joints (Fig. 3b) [4].

a)

b) c) 

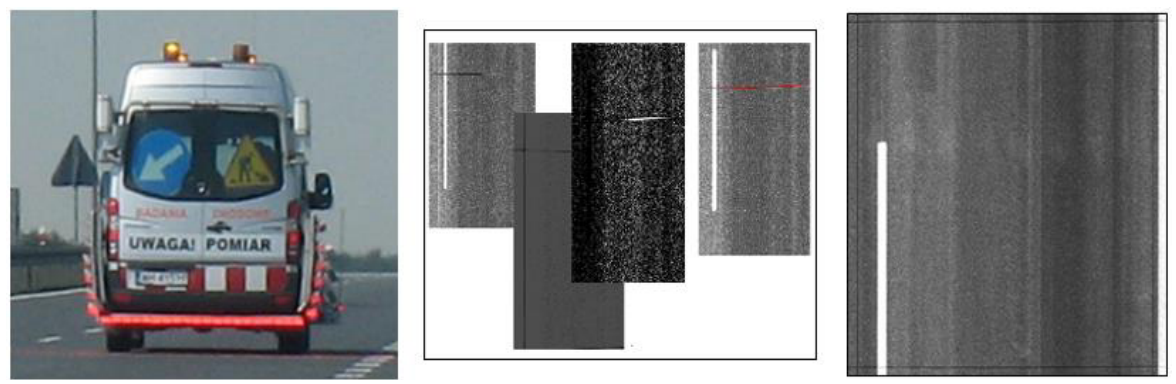

Fig. 3. The process of collecting the data representing the surface conditions of concrete slabs-

Since the concrete slabs in the traffic lanes exceeded the maximum operating width of the instrument it was necessary to synchronise the data from adjacent passes. Then the data were merged to cover the whole concrete lane and to retrieve the respective images. For accurate analysis of the coded distresses, macro images were processed in such a way that a single image showed a single slab with straight vertical longitudinal edges which were used for coding the geometry of each distress. The images have a margin of $15-20 \mathrm{~cm}$ which enabled full evaluation of pavement distress, including longitudinal and transverse cracking, corner breaks, edge cracking, spalled joints, defective joint sealing, surface damage and patches.

The example slab (Fig. 3c) is $4 \mathrm{~m}$ wide by $5 \mathrm{~m}$ long and the image size is $1568 \mathrm{x}$ $2571 \mathrm{px}$. The slab layout map was used in the final step for quick verification of the field data.

\section{The use of analysis results in the concrete pavements management system}

The starting point for developing the functionality of an efficient concrete pavement distress evaluation system should be reliable, accurate and up-to-date information on the condition of pavement which is the fundamental basis of regular maintenance of road network. These data provide also the input for short and long-term planning of maintenance treatments. They give feedback on the effectiveness of different pavement treatment types and technologies. Moreover, they enable estimating further deterioration of the pavement and creating on this basis pavement deterioration models used in planning of expenditures in the long term.

Therefore, in response to the demand for a system that would provide easy access to the required data, it was necessary to create a consolidated database including display function that would enable easy loading of the digital imaging data (grouped by survey) and, in particular, identification of the surface distresses with layout map reference to be used for subsequent monitoring. The windows presented in Fig. 4 are mutually synchronised such that when a selected location is changed in one window, the contents of the remaining windows is changed accordingly. This enables combining and analysing in the chosen way the displayed information from the different surveys (both historic and current). 
The database consolidated in this way supports their further processing, including without limitation measuring the length, width and surface areas of the identified distresses. With these operations, the condition evaluation performed with such distress evaluation system is in compliance with the requirements of SOSN-B [5] and DSN [6] guidelines.

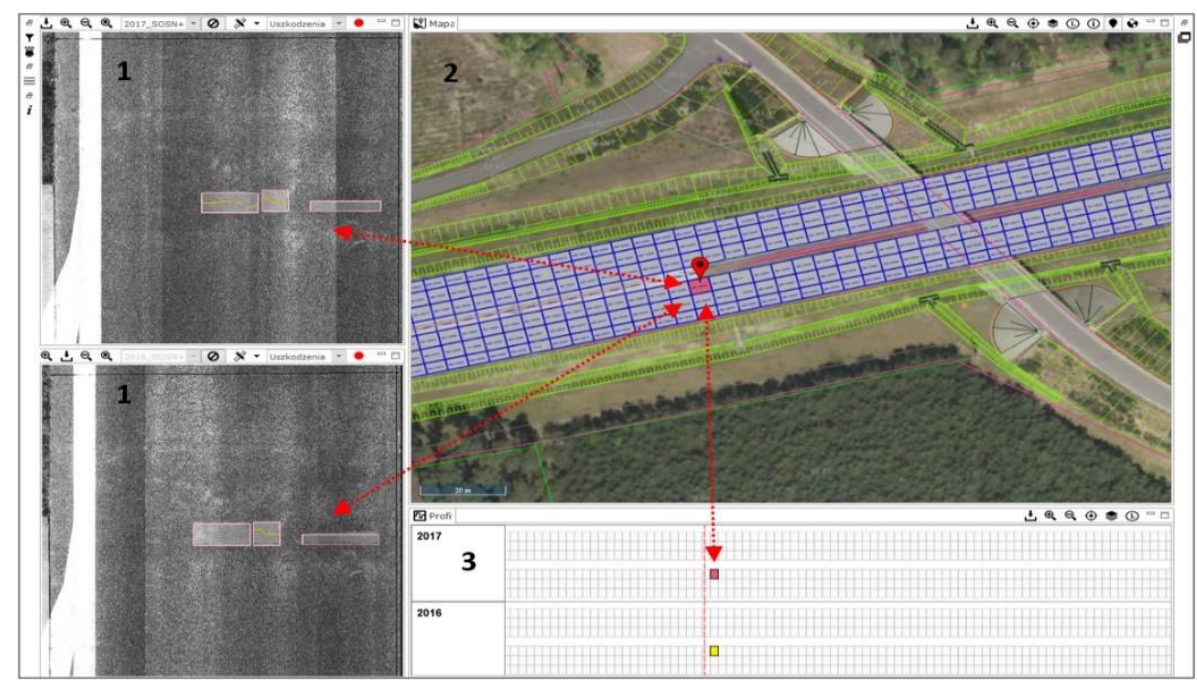

Fig. 4. An exemplary view of the concrete pavement management system window, 1. Macro photos of single concrete slabs in relation to a specific measuring campaign. 2. Geodetic map with marking and location area of all individual concrete slabs. 3. A thematic profile with appropriately defined data

Moreover, replacing the inspector's subjective assessment with objective evaluation of the distress geometry and size data (width, length, area) accompanied with display of values on the unit screen (note that this enables conducting case studies on large populations) makes it a reliable surveying method free of subjectivity related deficiencies typical of visual surveys. This approach enables also analysing the changes in the condition of pavement between surveys and after repairs for analytical and verification purposes (Fig. 5). 

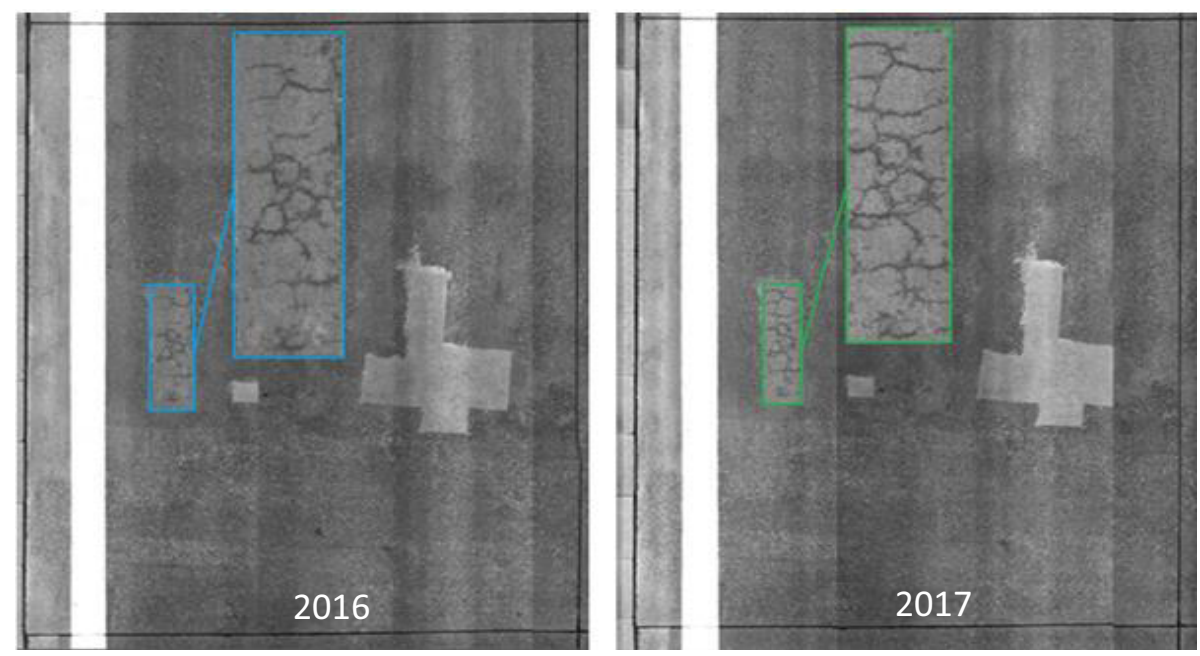

Fig. 5. View of the macro images of the same concrete slab for analysing the progress of changes to surface repairs in the subsequent surveys

\section{Conclusions}

Experimental aspect presented in this paper is a big step towards quality improvement in the concrete pavement management systems. With precise analyses at the individual concrete slab level (taking into account full scope of geometric data) and constant updating of databases with the data obtained through automated pavement condition imaging procedure it is possible not only to obtain "static information" but also, by introducing the time factor, to determine the rate of change of the pavement condition being the necessary input for modelling its residual life. The knowledge acquired in this way will directly influence the timing and type of response of the maintenance teams making it more efficient both in selection of maintenance treatments and in planning the maintenance policy itself.

The distress evaluation system designed in this way in relation to concrete pavements will provide, over a long-term period of observation a unique set of data which can be used for analyses (studying a large population, in this case more than 121 thousand slabs) which could not be done until recently.

What is important is that this concrete slabs management method will support decision making in relation to maintenance treatments and verification of their quality. Moreover, it will provide perfect data for predicting the deterioration models for each type of distress and the applied repair technique.

\section{References}

1. Glinicki M.A., Durability of concrete in road pavements. Effect of microstructure, materials design and diagnostics. IBDiM, Warszawa, 2011 
2. Concrete Pavement Preservation Guide, Second Edition, September 2014, FHWA Publication.

3. Sudyka J., Mechowski T., Harasim P.: Modern pavement diagnostic techniques in road network maintenance. IV International Conference „Modern Technologies in Highway Engineering”, Poznan 2009.

4. Bukowicki M., Opara K., Exact localization damages on concrete slab pavements., World Conference on Pavement and Asset Management, WCPAM2017, Milan, Italy - June 12/16, 2017.

5. Collective work: „Concrete Pavement Condition Assessment System”, SOSNB, General Directorate for National Roads and Highways (GDDKiA), Warsaw 2007.

6. Collective work: „Road pavement and pavement elements diagnostics”, DSN, General Directorate for National Roads and Highways (GDDKiA), Warsaw 2015. 